\title{
Gestaltungshinweise für die praktische Umsetzung der Gefährdungsbeurteilung psychischer Belastung
}

\author{
Kristin Gilbert ${ }^{1} \cdot$ Karolina A. Kirmse $^{1} \cdot$ Ulrike Pietrzyk $^{1} \cdot$ Anne Steputat-Rätze $^{1}$ \\ Online publiziert: 15 . April 2020 \\ (c) Der/die Autor(en) 2020
}

Schlüsselwörter Menschengerechte Arbeitsgestaltung · Betrieblicher Arbeits- und Gesundheitsschutz · Verhältnis- und Verhaltensprävention · Erfolgsbausteine für die Gefährdungsbeurteilung · Analyse psychischer Belastung

\section{Veränderungen in der Arbeitswelt - Agieren statt reagieren}

Weder in der Gesellschaft als Ganzem noch im Teilbereich der Erwerbsarbeit sind Fehlbeanspruchungen des Menschen und Gesundheitsrisiken bewältigt. Zwar sind Erfolge bei der Prävention von Berufskrankheiten und in Form der Reduzierung von Arbeitsunfällen zu verzeichnen (BAuA 2019; DGUV 2019), jedoch führen die kontinuierlichen Veränderungsprozesse in der Arbeitswelt zu neuen Belastungen.

Die Entwicklung hin zur Dienstleistungs- und Wissensgesellschaft sowie der technische Fortschritt gehen mit der Zunahme psychischer Arbeitsanforderungen einher. Der Fokus menschlicher Arbeit liegt zukünftig stärker in kognitiven, emotionalen und sozialen Anforderungen (Schütte und Rothe 2018). Dies führt zu geänderten Erfordernissen hinsichtlich der benötigten Qualifikation und Kompetenzen, sowie zu neuen Berufsbildern, Arbeitsaufgaben und -tätigkeiten. Zudem erfordert die Digitalisierung oftmals eine Neugestaltung von bestehenden Geschäfts- und Arbeitsprozessen (Hacker 2018a).

Die Verschärfung des Wettbewerbs durch die Verlagerung vom klassischen Kostenwettbewerb zum Wettbewerb um innovative und kundenwunschbezogene Produkte füh-

\footnotetext{
Kristin Gilbert

kristin.gilbert@tu-dresden.de

Karolina A. Kirmse

karolina.kirmse@tu-dresden.de

Dr. rer. nat. Ulrike Pietrzyk

ulrike.pietrzyk@tu-dresden.de

Anne Steputat-Rätze

anne.steputat-raetze@tu-dresden.de

1 Fakultät Psychologie, Arbeitsgruppe

Wissen-Denken-Handeln, Technische Universität Dresden, 01062 Dresden, Deutschland
}

ren dazu, dass sich für Unternehmen der Preis- und Kostendruck, Terminzwänge und die Notwendigkeit, ständig neue bzw. veränderte Produkte zu entwickeln und abzusetzen, verstärken (Zink und Bosse 2019; Eckert 2017). Infolgedessen ist eine Intensivierung der Arbeit zu beobachten, die durch zunehmende Flexibilisierung (in numerischer, funktionaler, räumlicher, vor allem aber zeitlicher Hinsicht) sowie einen erhöhten Zeit- und Leistungsdruck für die Mitarbeiterinnen und Mitarbeiter geprägt ist. Metaanalysen zeigen, dass insbesondere eine hohe Arbeitsintensität, ein geringer Handlungsspielraum, häufige Arbeitsunterbrechungen, Emotionsarbeit und mangelnde soziale Unterstützung mit einem erhöhten Risiko für psychische Beeinträchtigungen und negativen Folgen für die Sicherheit und Leistungsfähigkeit der Beschäftigten verbunden sind (z. B. Niedhammer et al. 2013; Rau und Buyken 2015; Rau und Henkel 2013; Rothe et al. 2017).

Seit 2013 wird im Arbeitsschutzgesetz die psychische Belastung explizit als mögliche Gesundheitsgefährdung bei der Arbeit benannt und die psychische Gesundheit als Zielgröße definiert (vgl. § 4 und $\S 5$ ArbSchG). Betriebe sind seither ausdrücklich aufgefordert, mit Hilfe des betrieblichen Arbeits- und Gesundheitsschutzes auch arbeitsbedingte psychische Gesundheitsgefahren zu verhüten, um die Sicherheit und den Gesundheitsschutz der Beschäftigten bei der Arbeit zu verbessern (vgl. ArbSchG, SGB VII).

Die Berücksichtigung psychischer Belastung im betrieblichen Arbeits- und Gesundheitsschutz bietet Betrieben die Chance, auf die aktuellen und zukünftigen Entwicklungstrends in der Arbeitswelt zu reagieren. Mittel- und langfristig kann die wirksame Prävention arbeitsbedingter Unfallund Gesundheitsgefahren zum Erhalt der Arbeitsfähigkeit von Beschäftigten beitragen, Fehlzeiten senken, die Produktivität erhöhen, die Attraktivität des Betriebs steigern und Fluktuation verhindern (vgl. Neuner 2019). Ein Weg zur Erreichung dieser Ziele ist die Gefährdungsbeurteilung psychischer Belastung. 
Der Beitrag soll Agierenden des Arbeits- und Gesundheitsschutzes Gestaltungshinweise für eine erfolgreiche Durchführung der Gefährdungsbeurteilung psychischer Belastung geben. Ausgehend von wissenschaftlichen Erkenntnissen und praktischen Erfahrungen werden Möglichkeiten aufgezeigt, um den Nutzen der Gefährdungsbeurteilung psychischer Belastung zu maximieren.

\section{Im Spannungsfeld zwischen gesetzlichen Anforderungen und betrieblicher Praxis}

Das Thema Gefährdungsbeurteilung psychischer Belastung führt in Betrieben nicht selten zu Verunsicherung - sowohl bei den Verantwortlichen als auch unter den Beschäftigten. Neben der Klärung des arbeitswissenschaftlichen Verständnisses von „Psychischer Belastung“ stellt sich für die betriebliche Praxis die Frage, wie die Verpflichtung zur Gefährdungsbeurteilung nach dem Arbeitsschutzgesetz sachgerecht und effizient bewältigt werden kann und welche Rahmenbedingungen erforderlich sind, um eine erfolgreiche, ökonomisch tragfähige und nachhaltige Umsetzung im Betrieb zu gewährleisten.

Gemäß DIN EN ISO 10075-1 ist psychische Belastung allgemein die Gesamtheit aller äußeren Faktoren, die psychisch auf Arbeitende einwirken. Anders als im Alltagsverständnis ist psychische Belastung damit neutral zu verstehen und kann mit „Gesamtheit aller Arbeitsanforderungen“ übersetzt werden. Nach dem Arbeitsprogramm Psyche der Gemeinsamen Deutschen Arbeitsschutzstrategie (GDA 2018) sollen bei der psychischen Arbeitsbelastung fünf Merkmalsbereiche berücksichtigt werden: Die Arbeitsaufgaben (der Arbeitsinhalt), die Arbeitsorganisation, die sozialen Beziehungen, die Arbeitsumgebung und die Neuen Arbeitsformen. Zu beachten ist, dass eine einzige Veränderung zu Wirkungen innerhalb mehrerer Merkmalsbereiche führen kann. Beispielsweise führt die Einführung einer neuen Technologie oder Software nicht nur zu Veränderungen bei der Arbeitsplatzgestaltung. Neue Arbeitsmittel können zusätzlich auch Auswirkungen auf die Arbeitsaufgabe (z.B. auf die erforderliche Qualifikation für die Tätigkeit), die Arbeitsorganisation (z.B. veränderte Arbeitsabläufe, Arbeitszeiten und Pausen) und die sozialen Beziehungen (z.B. die Möglichkeit von Unterstützung zwischen Kolleginnen und Kollegen) haben.

Unter betrieblichen Arbeitsschutz-Agierenden taucht oft die Frage auf, ob und wie psychische Belastung erfasst oder gemessen werden kann. Grenzwerte, wie beispielsweise für Lärm, gibt es für psychische Belastung nicht. Stattdessen existieren Kriterien menschengerecht gestalteter Arbeit (vgl. u.a. DIN EN ISO 9241-2; DIN EN ISO 6385:2004; DIN EN ISO 10075-2). Sie definieren laut $\S 2$ ArbSchG den Soll- oder Zielzustand der Arbeitsgestaltung.
Menschengerechte Gestaltung beinhaltet, dass die Arbeit die physische und psychische Gesundheit und Leistungsfähigkeit weder kurz- noch langfristig beeinträchtigt, sondern bestenfalls stärkt. Die Arbeit soll ,,an die Bedürfnisse und Qualifikationen des arbeitenden Menschen angepasst sein und eine Entwicklung der Persönlichkeit sowie eine Entfaltung von Potentialen und Kompetenzen fördern“ (GfA 2016, S. 3; Hacker und Sachse 2014).

Das Gefährdungspotential der psychischen Belastung leitet sich, analog zum Gefährdungspotential physischer Belastung, aus den gesundheitsrelevanten Folgen ab, die während oder infolge der Ausübung der Tätigkeit entstehen (Rau und Buyken 2015). Arbeitsanforderungen, die mit negativen Fehlbeanspruchungsfolgen einhergehen und beispielsweise Gesundheitsbeeinträchtigungen hervorrufen, sind als Gefährdungen einzustufen.

Die Gefährdungsbeurteilung psychischer Belastung liefert die Grundlage dafür, dass im Falle von psychischen Gefährdungen wirksame Maßnahmen zur menschengerechten Gestaltung der Arbeit eingeleitet werden können. Die auf Grundlage der Gefährdungsbeurteilung abgeleiteten Maßnahmen zielen hauptsächlich auf eine Verbesserung der Arbeitsbedingungen ab (z. B. aufgabenbezogene und organisatorische Verbesserungen wie angemessene Handlungsund Entscheidungsspielräume, funktionierende Informationsflüsse, mitarbeiterorientierte Arbeitszeitgestaltung). Gleichzeitig sind die Beschäftigten mit personenbezogenen Maßnahmen (z.B. Stressbewältigungs- und Kommunikationstraining, Qualifizierung) in ihrer Kompetenz zu fördern. Der arbeitswissenschaftliche Grundsatz „,Verhältnisbezogene Maßnahmen gehen vor verhaltensbezogenen Maßnahmen“ ist dabei zu berücksichtigen. Im Arbeitsschutzgesetz ist verankert: „Gefahren sind an ihrer Quelle zu bekämpfen“ und „Individuelle Schutzmaßnahmen sind nachrangig zu anderen Maßnahmen“ ( $\$ 4$ Abs. 5 ArbSchG).

Die Gefährdungsbeurteilung psychischer Belastung fokussiert somit nicht die personenbezogenen gesundheitlichen Auswirkungen, sondern setzt an den Bedingungsfaktoren (den Arbeitsanforderungen) an. Auf diese Weise sollen Gesundheitsgefährdungen am Arbeitsplatz frühzeitig erkannt und konkrete Präventionsmaßnahmen zur Reduktion bzw. zur Vermeidung abgeleitet werden.

Der Schutz der Beschäftigten vor psychischen Gefährdungen und ihren Folgen ist nicht nur eine gesetzliche und ethisch-moralische Verpflichtung der Arbeitgeberin bzw. des Arbeitgebers, sondern kann als betriebswirtschaftlicher Vorteil genutzt werden.

Investitionen in die Gefährdungsbeurteilung psychischer Belastung im Rahmen des betrieblichen Arbeitsund Gesundheitsschutzes ,,rechnen“ sich für die Unternehmen. Durch die Verringerung psychischer Fehlbelastungen können Kosten für Arbeitsunfälle und arbeitsbedingte Erkrankungen reduziert sowie Ausfallzeiten und Störungen 
der Betriebsabläufe minimiert werden (vgl. Eichhorn und Schuller 2017). Hinzu kommt ein Wertzuwachs durch gestiegene Motivation und Zufriedenheit der Beschäftigten sowie durch ein verbessertes Unternehmensimage. Der Nettonutzen des gesamten Arbeits- und Gesundheitsschutzes für Unternehmen lässt sich mit einem Return on Prevention (ROP) angegeben. Mit einem geschätzten ROP von 2,20 zahlt sich jeder investierte Euro für das Unternehmen in den Arbeits- und Gesundheitsschutz mit 2,20€ aus (Bräunig und Kohstall 2013; iga 2015).

Ungeachtet der hohen Verbreitung psychischer Fehlbelastung (z.B. Lohmann-Haislah 2012) sowie deren negativen Auswirkungen auf die Gesundheit und Leistungsfähigkeit von Beschäftigten (Rothe et al. 2017; Rau und Buyken 2015; Paridon 2016) wird den gesetzlichen Arbeitgeberverpflichtungen bislang nur unzureichend nachgekommen (Beck und Lenhardt 2019; European Agency for Safety and Health at Work 2016). Die Potentiale der Gefährdungsbeurteilung psychischer Belastung werden somit nicht ausgeschöpft.

Beck und Lenhardt (2019) ermittelten in einer repräsentativen Befragung bei deutschen Unternehmen, dass nur $21 \%$ der Betriebe die psychischen Gefährdungen in ihrer Gefährdungsbeurteilung berücksichtigen. Diese Quote ist vergleichbar mit den Ergebnissen der GDA-Betriebs- und Beschäftigtenbefragungen aus den Jahren 2015 und 2011, wonach lediglich $22 \%$ der Betriebe eine Gefährdungsbeurteilung psychischer Belastung vornahmen (Geschäftsstelle der Nationalen Arbeitsschutzkonferenz 2016). Im internationalen Vergleich liegt die Umsetzungsrate in Deutschland lediglich im unteren Mittelfeld (DGPPN-Studie, Hofmann 2014).

Das BAuA-Projekt „Psychische Belastung in der betrieblichen Praxis“ (Lenhardt 2017) zeigt auf, dass die Ursachen für die geringe flächendeckende Umsetzung der Gefährdungsbeurteilung psychischer Belastung in großen Handlungsunsicherheiten begründet liegen. Neben der Auswahl eines geeigneten Instruments zur Ermittlung von psychischer Belastung stehen Betriebe vor den Fragen, wie die ermittelten Gefährdungen bewertet, angemessene Maßnahmen abgeleitet und die Wirkung dieser Maßnahmen kontrolliert werden sollen (Beck und Lenhardt 2019). Auch die Gefährdungsbarometer ${ }^{\circledR}$-Studie des EO-Instituts macht deutlich, dass mangelndes Wissen und Umsetzungsprobleme hinsichtlich der Gefährdungsbeurteilung psychischer Belastung bestehen (EO-Institut 2015).

\section{Handlungsempfehlungen für betriebliche Agierende}

Damit Betriebe vom Nutzen der Gefährdungsbeurteilung psychischer Belastung profitieren können, werden im Fol- genden ausgewählte Handlungsempfehlungen für eine erfolgreiche Umsetzung der Gefährdungsbeurteilung psychischer Belastung gegeben. Dabei werden jene Aspekte der Gefährdungsbeurteilung psychischer Belastung fokussiert, die sich in Betrieben als Stolpersteine erweisen haben.

Methodisches Vorgehen bei der Ableitung der Handlungsempfehlungen Für die Ableitung der Handlungsempfehlungen wurden Ergebnisse und Erfahrungen aus verschiedenen Forschungs-, Praxis- und Transferprojekten der Arbeitsgruppe Wissen-Denken-Handeln an der Technischen Universität Dresden integriert ${ }^{1}$. Die Erkenntnisse stammen von zahlreichen Gefährdungsbeurteilungen psychischer Belastung, die in verschiedenen Branchen und unterschiedlichsten Betriebsgrößen (bis 10 Beschäftigte, 10 bis $50 \mathrm{Be}$ schäftigten und mehr als 50 Beschäftigte) im Zeitraum 2012 bis 2019 durchgeführt wurden. Die Gefährdungsbeurteilungen wurden mittels Begehungen (Beobachtungsinterviews), schriftlichen Befragungen und Workshops von der Arbeitsgruppe entweder eigens ausgeführt oder wissenschaftlich begleitet und evaluiert. Bei der Ableitung der Handlungsempfehlungen fanden Tätigkeiten aus dem Handel, dem Bildungswesen, der öffentlichen Verwaltung, dem Finanzund Versicherungswesen, dem Sozialversicherungswesen sowie aus der Pflege Berücksichtigung. Durch die Diversität der untersuchten Branchen und Tätigkeiten wird eine Übertragung der Handlungsempfehlungen auf ein breites Anwendungsfeld ermöglicht.

Die Datenerhebung erfolgte mit standardisierten branchenspezifischen Instrumenten für die Gefährdungsbeurteilung psychischer Belastung. Neben Beobachtungsinterviews (BAGAB: Hubrich 2011; TA-DD: Looks et al. 2011; SAG: Debitz et al. 2014) und Mitarbeiterbefragungen (SABiA: Schmidt et al. 2012; Prüfliste: Mühlpfordt und Richter 2003) kamen die Instrumente aus dem PegAProgramm (PegA - Psychische Belastung erfassen, gesunde Arbeit gestalten: BGHW 2019) sowie das Verfahren „Gute Stationsorganisation“ (Stab und Hacker 2016) zur Anwendung. Der Ablauf der Gefährdungsbeurteilungen orientierte sich in den Betrieben stets an den ,Sieben Schritten zur Gefährdungsbeurteilung“ der BAuA (2020; Abb. 2).

Im Rahmen des Projekt- und Qualitätsmanagements sowie zur Evaluation der Gefährdungsbeurteilung in den Betrieben wurden detaillierte Informationen über die Art und Weise der Umsetzung der Gefährdungsbeurteilungen gesammelt. Um aus den gewonnenen Informationen allgemeingültige Handlungsempfehlungen für eine erfolgreiche Umsetzung einer Gefährdungsbeurteilung abzuleiten, wur-

\footnotetext{
$\overline{1}$ Wir möchten uns an dieser Stelle bei allen Praxispartnerinnen und -partnern bedanken, die uns bei der Durchführung der Untersuchungen zum Themenbereich Gefährdungsbeurteilung psychischer Belastung sowohl inhaltlich als auch organisatorisch unterstützt haben.
} 


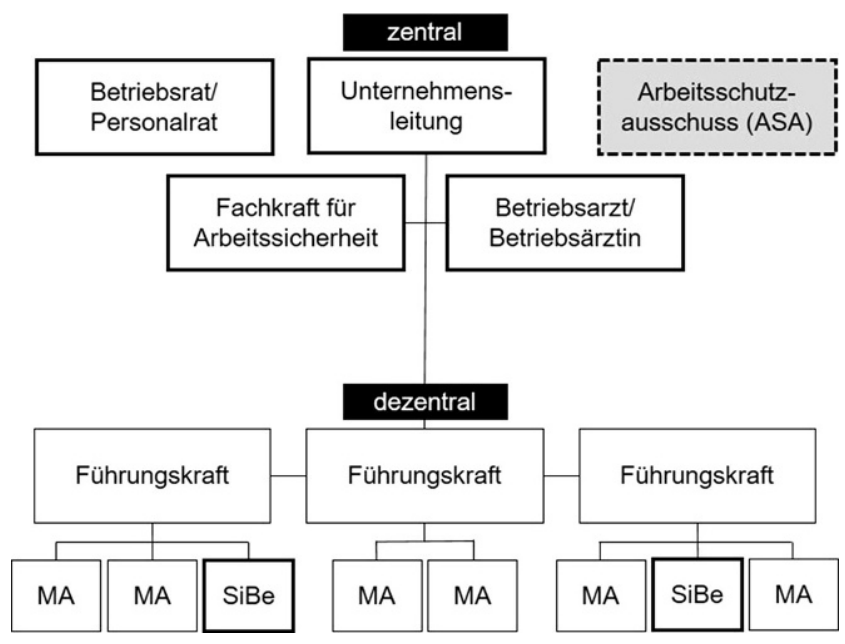

Abb. 1 Zentral und dezentral organisierte Gefährdungsbeurteilung psychischer Belastung. (Die Mitglieder des Arbeitsschutzausschusses sind mit einem dunklen Rahmen gekennzeichnet. SiBe $=$ Sicherheitsbeauftragte)

den die Erkenntnisse qualitativ aufgearbeitet und das umgesetzte Vorgehen gemeinsam mit den betrieblichen Agierenden reflektiert. Parallel hierzu fand in der Arbeitsgruppe eine deduktiv-induktive Analyse von ,typischen Problemfeldern" und Best-Practices bei der Gefährdungsbeurteilung psychischer Belastung statt. Auf diese Weise konnten Erfolgsbausteine identifiziert sowie entsprechende Handlungsempfehlungen abgeleitet werden.

\subsection{Organisation - Verantwortliche, Tätigkeiten und Methoden festlegen}

Entscheidend für den Erfolg der Gefährdungsbeurteilung ist eine systematische und strukturierte Planung und Vorbereitung. Die praktische Erfahrung in verschiedenen Betrieben zeigt, dass insbesondere die Festlegung von Verantwortlichkeiten, die Bestimmung von Tätigkeitsbereichen und die Auswahl einer geeigneten Methode häufige Herausforderungen bei der Organisation der Gefährdungsbeurteilung darstellen. Daher werden nachfolgend Hinweise gegeben, wie Betriebe ihre Gefährdungsbeurteilung hinsichtlich dieser drei Aspekte zielführend organisieren können.

Festlegung der Verantwortlichen für die Umsetzung Grundsätzlich liegt die Verantwortung für die Umsetzung der Gefährdungsbeurteilung bei der Arbeitgeberin oder dem Arbeitgeber ( $\$ 3$ ArbSchG). Die Arbeitgeberin oder der Arbeitgeber kann zuverlässige und fachkundige Personen damit beauftragen, ihre oder seine Aufgaben in eigener Verantwortung wahrzunehmen ( $\$ 7$ ArbSchG). Es ist wichtig, bei der Pflichtenübertragung die Verantwortungs- und Aufgabenbereiche aller Funktionsträgerinnen und -träger im Arbeitsschutz sowie die erforderlichen
Kompetenzen konkret, umfassend und vor allem schriftlich festzulegen. Die Arbeitgeberin oder der Arbeitgeber bleibt jedoch verantwortlich für die Aufsicht und Kontrolle und hat dafür zu sorgen, dass die übertragenen unternehmerischen Pflichten auch tatsächlich umgesetzt werden (GDA 2017a).

Für die fachliche Beratung und Unterstützung der Unternehmensleitung sind gesetzlich die Fachkraft für Arbeitssicherheit (Sifa) und die Betriebsärztin oder der Betriebsarzt vorgesehen ( $\S 3$ und 6 Arbeitssicherheitsgesetz - ASiG; GDA 2016). Zusätzlich ist das Einbeziehen von weiteren Arbeitsschutz-Agierenden wie den Sicherheitsbeauftragten (§ 22 SGB VII) oder der Schwerbehindertenvertretung (§ 178 SGB IX) sinnvoll.

Es sollte für die Gefährdungsbeurteilung psychischer Belastung ein paritätisch besetzter Steuerkreis gebildet werden. Der Arbeitsschutzausschuss (ASA), den Unternehmen mit mehr als 20 Beschäftigten gemäß $§ 11$ Arbeitssicherheitsgesetz (ASiG) bilden müssen, bietet sich als übergeordnetes betriebliches Koordinations- und Beratungsgremium an (Abb. 1).

Hinsichtlich der Festlegung von Verantwortlichkeiten stellt sich in Betrieben die grundsätzliche Frage, ob die Gefährdungsbeurteilung zentral oder dezentral organisiert werden soll.

Bei einer zentral organisierten Gefährdungsbeurteilung liegt das Aufgaben- und Verantwortungsspektrum bei der Unternehmensleitung oder bei einer zentralen Organisationseinheit, zum Beispiel dem zentralen Arbeits- und Gesundheitsschutz oder der Personalabteilung. Die Gefährdungsbeurteilung wird durch die Unternehmensleitung selbst oder durch die zentrale Einheit für alle lokalen Unternehmenseinheiten (Standorte, Filialen, Geschäftsstellen o. Ä.) durchgeführt („Gefährdungsbeurteilung von oben“).

Bei der dezentral organisierten Gefährdungsbeurteilung wird die Verantwortung von der Geschäftsführung auf die lokalen Geschäftsleitungen und gegebenenfalls die Steuerkreise vor Ort übertragen. Die lokalen Geschäftsleitungen werden bei ihren Aufgaben durch zentrale Einheiten unterstützt, beraten und erforderlichenfalls qualifiziert (,Gefährdungsbeurteilung von unten“).

Die Frage, welche der beiden Möglichkeiten die Geeignetere ist, kann nicht eindeutig beantwortet werden und ist von den Gegebenheiten im Betrieb abhängig. Ein Vorteil der zentral organisierten Gefährdungsbeurteilung liegt in der Fachkompetenz der Zentrale bzw. in einem überschaubaren Qualifikationsaufwand für die begrenzte Anzahl verantwortlicher zentraler Personen. Zudem kann ein einheitlicher Standard bei der Gefährdungsbeurteilung gesichert werden und das Unternehmen behält über das Vorgehen und die Ergebnisse einen guten Überblick.

Vorteile der „dezentralen Variante“ sind, dass die dezentralen Führungskräfte meist umfassende Kenntnisse von 
den Arbeitsbedingungen und den Gegebenheiten vor Ort besitzen. Das Wissen kann das Ableiten geeigneter Maßnahmen erleichtern. Da die dezentralen Führungskräfte in Entscheidungen und in den Prozess eingebunden werden, ist deren Commitment und die Motivation erfahrungsgemäß höher als wenn ,fremde“ Unternehmenseinheiten die Gefährdungsbeurteilung für ihren Bereich übernehmen.

Unabhängig der gewählten Organisationsstruktur ist es wichtig, dass die Gefährdungsbeurteilung psychischer Belastung strukturell fest im Betrieb verankert wird, damit sie nachhaltig wirken kann.

\section{Zusammenfassen von Tätigkeitsbereichen oder Arbeitsplät-}

zen zu Gruppen Eine gute Nachricht für Betriebe ist, dass die Gefährdungsbeurteilung nicht für jeden Arbeitsplatz von allen Beschäftigten durchgeführt werden muss. Vielmehr dürfen Tätigkeiten mit gleichartigen Arbeitsbedingungen zu einer Gruppe zusammengefasst werden. Es ist ausreichend, aus der Gruppe gleichartiger Tätigkeiten nur einen Arbeitsplatz oder eine Tätigkeit zu analysieren ( $\$ 5$ Abs. 2 ArbSchG).

Das Zusammenfassen von gleichartigen Tätigkeiten zu Gruppen ist nicht nur ein wichtiger Schritt zur Aufwandsreduktion, sondern bestimmt maßgeblich die Qualität der Ergebnisse. Es ist daher lohnenswert, die Einteilung der zu untersuchenden Einheiten in der Planungsphase der Gefährdungsbeurteilung sorgfältig vorzunehmen.

Es ist wichtig, dass die Festlegung der zu betrachtenden Gruppen tätigkeitsbezogen erfolgt. Nach einer Auflistung aller Tätigkeiten im Betrieb muss geprüft werden, inwieweit diese vergleichbar oder verschieden sind. Dabei gilt die Regel: Gleichartige Tätigkeiten dürfen zusammengefasst, verschiedenartige müssen jede für sich betrachtet werden.

Die tätigkeitsbezogene Festlegung der Analyseeinheiten bei der Gefährdungsbeurteilung psychischer Belastung kann auch bedeuten, dass andere Gruppen als bei der ,klassischen“ Gefährdungsbeurteilung betrachtet werden. Beispielsweise können Bildschirmarbeitsplätze in der „klassischen“ Gefährdungsbeurteilung als eine Einheit analysiert werden. Dies ist bei der Gefährdungsbeurteilung psychischer Belastung nicht zweckmäßig, da Bildschirmarbeitsplätze sehr unterschiedliche Arbeitsanforderungen haben können. So sind die Bildschirmarbeitsplätze von Sachbearbeitenden, Personalverantwortlichen oder Führungskräften nicht vergleichbar, da sie sich hinsichtlich ihrer Arbeitsaufgaben und ihrer Arbeitsorganisation teilweise stark unterscheiden. Die Tätigkeit mehrerer Sachbearbeitender in einem bestimmten Sachgebiet oder aller Personalverantwortlichen in der Personalabteilung können dagegen zumeist als eine Gruppe betrachtet werden, insofern sie im jeweiligen Betrieb vergleichbar sind.

Betriebe können die Aufbau- und Ablauforganisation bzw. ihr Organigramm als sinnvolle Unterstützung bei der
Festlegung der zu betrachtenden Gruppen nutzen. Zusätzlich müssen die Gruppen tätigkeitsbezogen geprüft werden, da sonst das Risiko fehlerhafter Ergebnisse besteht. Beispielsweise wurden in einer Einzelhandelsfiliale entsprechend der Filialorganisation sechs Tätigkeitsbereiche unterschieden (u.a. Warenverräumung, Kassieren, Dekoration). Der Abwechslungsreichtum innerhalb der Bereiche wurde als gering eingestuft. Es wurde allerdings außer Acht gelassen, dass die Beschäftigten bereichsübergreifend eingesetzt wurden. Tatsächlich lag für die Gruppe der Verkäuferinnen und Verkäufer somit ein hoher Abwechslungsreichtum vor.

Hinsichtlich der Festlegung der Gruppen soll zudem darauf hingewiesen werden, dass verschiedenartige Tätigkeiten auch nicht zusammengefasst werden dürfen, um zum Beispiel den Aufwand für die Beurteilung zu reduzieren oder die Anonymität bei ansonsten zu geringen Teilnahmezahlen zu sichern. So wurden beispielsweise in einem Unternehmen Mitarbeiterbefragungen aus verschiedenen Unternehmensbereichen mit geringen Beschäftigtenzahlen (z.B. Verwaltung, Führungskräfte, Reinigungskräfte) zu einer Gruppe „Weitere“ zusammengefasst. Dieses Vorgehen widerspricht der gesetzlichen Verpflichtung nach einer tätigkeitsbezogenen Gefährdungsbeurteilung gemäß $§ 5$ Abs. 2 ArbSchG. Des Weiteren entsteht ein undifferenziertes Bild, aus dem weder ein sinnvolles Ergebnis noch Maßnahmen abgeleitet werden können.

Auswahl einer oder mehrerer Methoden In Betrieben bestehen häufig Unsicherheiten, welche Methode für die Gefährdungsbeurteilung psychischer Belastung eingesetzt werden soll, da keine gesetzlichen Vorgaben existieren.

Generell haben sich drei methodische Ansätze etabliert, die einzeln oder kombiniert eingesetzt werden können: die schriftliche Mitarbeiterbefragung, die Beobachtung bzw. das Beobachtungsinterview durch eine fachkundige Person (Begehung) und der moderierte Workshop (GDA 2017b). Jede Methode ist auf unterschiedliche Rahmenbedingungen ausgerichtet und geht mit verschiedenen Vor- und Nachteilen einher. Die Auswahl muss daher stets vor dem Hintergrund der spezifischen Gegebenheiten, Erfahrungen und Kompetenzen im Betrieb erfolgen. Wichtig ist, die richtige Methode für jede einzelne Tätigkeit zu finden. Es ist nicht sinnvoll, pauschal für alle Tätigkeiten im Unternehmen dieselbe Methode zu wählen.

Im Rahmen der durchgeführten Projekte wurde deutlich, dass manche Unternehmen ihre Methode eher ,intuitiv“ und wenig vorausschauend auswählen. Wenn jedoch beispielsweise ein unternehmensübergreifender Fragebogen eingesetzt wird, obwohl die Anforderung an die Mindestteilnehmerzahl in bestimmten Abteilungen nicht erfüllt ist, kann dadurch die Teilnahmebereitschaft der Beschäftigten sinken und die Qualität der Gefährdungsbeurteilung beeinträchtigt 
werden. Die Anwendungsvoraussetzungen einer Methode sollten deshalb stets vor dem Einsatz geprüft werden.

Ausgehend von betrieblichen Erfahrungen haben sich folgende Aspekte bei der Auswahl einer geeigneten Methode als die wichtigsten Entscheidungskriterien herausgestellt:

1. Anzahl der Beschäftigten: Befragungen sind an Mindestteilnehmeranzahlen gebunden, um aussagekräftige und anonyme Ergebnisse zu erhalten. Auch für die Durchführung von Workshops ist eine Mindestanzahl an Teilnehmerinnen und Teilnehmern erforderlich. Relevant ist die Anzahl der Beschäftigten, die die zu betrachtende Tätigkeit ausführen, nicht die Größe der Gesamtbelegschaft. Die nachträgliche Zusammenfassung von Abteilungen, um die Mindestteilnehmerzahl zu erreichen, empfiehlt sich nicht (vgl. Abschnitt „Zusammenfassen von Tätigkeitsbereichen oder Arbeitsplätzen zu Gruppen“).

2. Personalkapazität und -planung: Des Weiteren muss die Personalkapazität und -planung bei der Auswahl der Methode berücksichtigt werden. Für die Zeit der Gefährdungsbeurteilung psychischer Belastung müssen Beschäftigte freigestellt und gegebenenfalls von Kolleginnen und Kollegen vertreten werden. Dies betrifft in stärkerem Ausmaß Befragungen und Workshops und in geringerem Ausmaß Begehungen.

3. Partizipation von Beschäftigten: Der Wunsch vieler Unternehmen ist es, die Beschäftigten in die Analyse, Bewertung und Gestaltung ihrer Arbeitsplätze einzubeziehen. Dies ermöglichen insbesondere Befragungen und Workshops.

4. Ableitung von Maßnahmen: Workshops eignen sich in besonderer Weise, wenn der Wunsch besteht, mit der Methode die Tätigkeit nicht nur zu analysieren und zu bewerten, sondern direkt auch Verbesserungsmaßnahmen abzuleiten.

Nachdem die Entscheidung für eine Methode getroffen wurde, folgt die Wahl eines konkreten Instrumentes zur Erfassung psychischer Belastung. Um aussagekräftige Ergebnisse zu erhalten, sollte das gewählte Instrument wissenschaftlich fundiert und validiert sein (entsprechend den Qualitätsgrundsätzen der Gemeinsamen Deutschen Arbeitsschutzstrategie; vgl. GDA 2018). Das Entwickeln eines neuen Instruments ,auf eigene Faust“ oder das Ändern bestehender Instrumente, z. B. durch das Einfügen oder Entfernen von Fragen, hat sich aus Qualitäts- und Kostengründen nicht bewährt. Einen Überblick über die zahlreichen Instrumente zur Ermittlung von psychischer Belastung finden sich beispielsweise bei Böckelmann und Seibt (2011) und Treier (2019). Auch die Berufsgenossenschaften und Unfallkassen stellen ihren Mitgliedsbetrieben geeignete branchenspezifische Instrumente für die Gefährdungsbeurteilung psychischer Belastung bereit.
Um zusätzliche Sicherheit über die Wahl der richtigen Methode oder des richtigen Instruments zu gewinnen, empfiehlt sich eine Pilot-Durchführung für ausgewählte Tätigkeiten. So kann geprüft werden, ob das Instrument passend und das Vorgehen stimmig ist.

\subsection{Partizipation - Beschäftigte und Führungskräfte beteiligen}

Um einen wirklichen Mehrwert für Beschäftigte und Unternehmen zu erreichen, müssen alle im Unternehmen vom Nutzen der Gefährdungsbeurteilung psychischer Belastung überzeugt sein. Oftmals sind neue Prozesse jedoch mit Widerstand gegen Veränderungen verbunden (,Das ist bei uns sowieso nicht umsetzbar“", etc.). Das bewährte Vorgehen zum Überwinden von Veränderungswiderstand ist die Beteiligung von Beschäftigten und Führungskräften an der Gefährdungsbeurteilung psychischer Belastung (partizipatives Vorgehen).

Bei der Gefährdungsbeurteilung geht es um die Arbeitsbedingungen. Dafür sind die Mitarbeiterinnen und Mitarbeiter die eigentlichen Expertinnen und Experten. Die Erfahrung zeigt, dass gemeinsam mit den Mitarbeiterinnen und Mitarbeitern die Gründe für die ermittelte Fehlbelastung zielführend eruiert und sinnvolle Maßnahmen abgeleitet werden können (vgl. Hacker 2018b). Gleichzeitig sichert die Partizipation eine hohe innerbetriebliche Akzeptanz des Vorgehens. Partizipation bezieht sich dabei nicht nur auf die aktive Mitwirkung an den einzelnen Schritten der Gefährdungsbeurteilung psychischer Belastung, sondern auch die rechtzeitige und umfassende Kommunikation im Betrieb (vgl. Abschn. 3.3).

\subsection{Kommunikation - Wissen und Vertrauen schaffen}

Als wichtige Stellschraube für eine erfolgreiche Umsetzung der Gefährdungsbeurteilung psychischer Belastung erweist sich in der betrieblichen Praxis eine umfassende Kommunikation. Eine transparente und vertrauensbildende Kommunikation stellt die Voraussetzung dafür dar, dass alle Beteiligten am Prozess mitwirken und offen und aufrichtig Auskunft geben. Auf diese Weise kann ein realistisches Bild von den Arbeitsbedingungen entstehen und es können gemeinsam wirkungsvolle Maßnahmen entwickelt werden. Insbesondere wertschätzende und adressatengerechte Vorabinformationen schaffen die erforderliche Wissens- und Vertrauensgrundlage für den weiteren Prozess.

Die Beschäftigten möchten wissen, warum und wie die Gefährdungsbeurteilung psychischer Belastung durchgeführt wird und welchen Mehrwert sie davon haben. Gleichzeitig ist es von Bedeutung, unangemessene Erwartungen zu vermeiden, indem thematisiert wird, was die Gefähr- 
dungsbeurteilung nicht leisten kann. Beispielsweise ist es zumeist nicht möglich, in einem einmaligen Workshop für sämtliche identifizierten psychischen Gefährdungen geeignete Verbesserungsmaßnahmen zu entwickeln.

Ein wichtiges Erfolgskriterium, um Ängsten und Missverständnissen vorzubeugen und um eine hohe Teilnahmemotivation zu sichern, ist das Informieren über die Bedingungsbezogenheit der Gefährdungsbeurteilung psychischer Belastung. Grundlegend ist, über den gesamten Prozess hinweg wiederholt zu betonen, dass es bei der Gefährdungsbeurteilung nicht um den psychischen Zustand einzelner Personen, sondern um eine Beurteilung der Arbeitsanforderungen geht.

Weiterhin wird der Schutz der Anonymität von den Beschäftigten als wichtiges Thema benannt. Insbesonders, wenn problematische Arbeitsbedingungen behandelt werden, wünschen sich Beschäftigte Vertraulichkeit. Es ist jedoch zu beachten, dass weder Workshops noch Begehungen vollständig anonym durchgeführt werden können. Die Erfahrung zeigt außerdem, dass Beschäftigte, die Nachteile befürchten oder Angst vor negativen Folgen haben, auch in einer anonymen Mitarbeiterbefragung nicht offen und ehrlich (oder gar nicht) antworten.

Vertrauen ist der Grundstein einer erfolgreichen Gefährdungsbeurteilung, in der Ressourcen und auch problematische Arbeitsbedingungen angesprochen werden können. Damit Beschäftigte sich öffnen, ist es wichtig, dass Betriebe die Grundsätze der Freiwilligkeit und Transparenz einhalten. Gleichzeitig ist eine Vertrauens- und Feedbackkultur im Unternehmen erforderlich, die von wechselseitigem Respekt geprägt ist und in der die Beschäftigte hierarchie- und funktionsübergreifend offen miteinander kommunizieren. Eine solche Unternehmenskultur sichert den Erfolg der Gefährdungsbeurteilung psychischer Belastung. Darüber hinaus steigert sie bei den Beschäftigten die Identifikation mit ihrem Unternehmen und erhöht die Arbeitgeberattraktivität.

\subsection{Qualifikation - Wissen und Kompetenzen sicherstellen}

Aufgrund der Möglichkeit für die Arbeitgeberin oder den Arbeitgeber, Pflichten bei der Gefährdungsbeurteilung psychischer Belastung an zuverlässige und fachkundige Personen zu übertragen, werden in der Praxis häufig Fachkräfte für Arbeitssicherheit sowie Sicherheitsbeauftragte mit der Durchführung der Gefährdungsbeurteilung betraut. Diese Personen verfügen oftmals über vorrangig technisch ausgerichtete Qualifikationen. Somit ist die Frage „Was versteht man unter psychischer Belastung und wie misst bzw. erfasst man sie?" in vielen Betrieben nicht leicht zu beantworten. Die fachgerechte Nutzung der verschiedenen Methoden zur Durchführung der Gefährdungsbeurteilung psychischer Be- lastung (vgl. Abschn. 3.1) und die Anwendung entsprechender Instrumente stellt viele betriebliche Arbeitsschutz-Agierende vor große Herausforderungen. Weiterhin zeigt sich, dass es häufig die betriebsspezifischen Rahmenbedingungen sind, welche die Erhebung der psychischen Belastung sowie die Maßnahmenableitung erschweren (z.B. saisonal variierendes Arbeitsaufkommen, durch technische Anlagen vorgegebenes Arbeitstempo).

Vor diesem Hintergrund ist es empfehlenswert, (externe) fachliche Expertinnen und Experten, die umfangreiches Wissen zur Gefährdungsbeurteilung psychischer Belastung besitzen (bspw. Arbeitspsychologinnen und Arbeitspsychologen, Arbeitswissenschaftlerinnen und Arbeitswissenschaftler oder Beraterinnen und Berater der Berufsgenossenschaften), unterstützend in die Durchführung der Gefährdungsbeurteilung einzubeziehen.

Zudem sollten durch eine Weiterqualifizierung von internen Arbeitsschutz-Agierenden die Kompetenzen zum Thema Gefährdungsbeurteilung psychischer Belastung (weiter) ausgebaut werden. Diese Empfehlung findet sich in der Broschüre „Empfehlungen zur Qualifizierung betrieblicher Akteure für die Umsetzung der Gefährdungsbeurteilung psychischer Belastung“ der Gemeinsamen Deutschen Arbeitsschutzstrategie wieder (GDA 2016). Eine Weiterqualifizierung kann beispielsweise im Rahmen von Schulungen erfolgen, in denen durch externe Trainerinnen oder Trainer zunächst ein einheitliches Begriffsverständnis geschaffen (vgl. Abschn. 3.3 - bedingungsbezogene Beurteilung der Arbeitsbedingungen) und Basiswissen zur Gefährdungsbeurteilung psychischer Belastung vermittelt wird. Weiterführend ist der Blick auf erfolgreiche Praxisbeispiele aus anderen Unternehmen zur Umsetzung der Gefährdungsbeurteilung sinnvoll.

Wird als Methode für die Gefährdungsbeurteilung psychischer Belastung ein moderierter Workshop eingesetzt, stellt die Qualifikation und Erfahrung der Workshop-Moderatorinnen und Moderatoren einen wichtigen Faktor für eine erfolgreiche Gefährdungsbeurteilung dar. In Unternehmen, die die Workshop-Methode nutzen, werden die Moderatorinnen und Moderatoren überwiegend intern oder extern geschult, anderenfalls greifen Moderatorinnen und Moderatoren auf anderweitig erworbene Erfahrungen zurück (z.B. auf Moderationsseminare im Studium). Spezifische Moderationsschulungen in Vorbereitung auf die Gefährdungsbeurteilung psychischer Belastung erwiesen sich als empfehlenswert, um die Workshops strukturiert, effizient und zielführend durchführen zu können. Eine qualifizierte Moderation ist insbesondere bei ,heiklen“ Themen, die im Workshop zur Sprache kommen (z. B. Führungskräftequalifikation und -verhalten), sowie in anspruchsvollen Situationen wichtig (z.B. Diskussion strukturieren, Beschäftige zum Thema zurückbringen). Zudem weisen betriebliche Arbeitsschutz-Agierende darauf hin, dass gut qualifizierte Mode- 


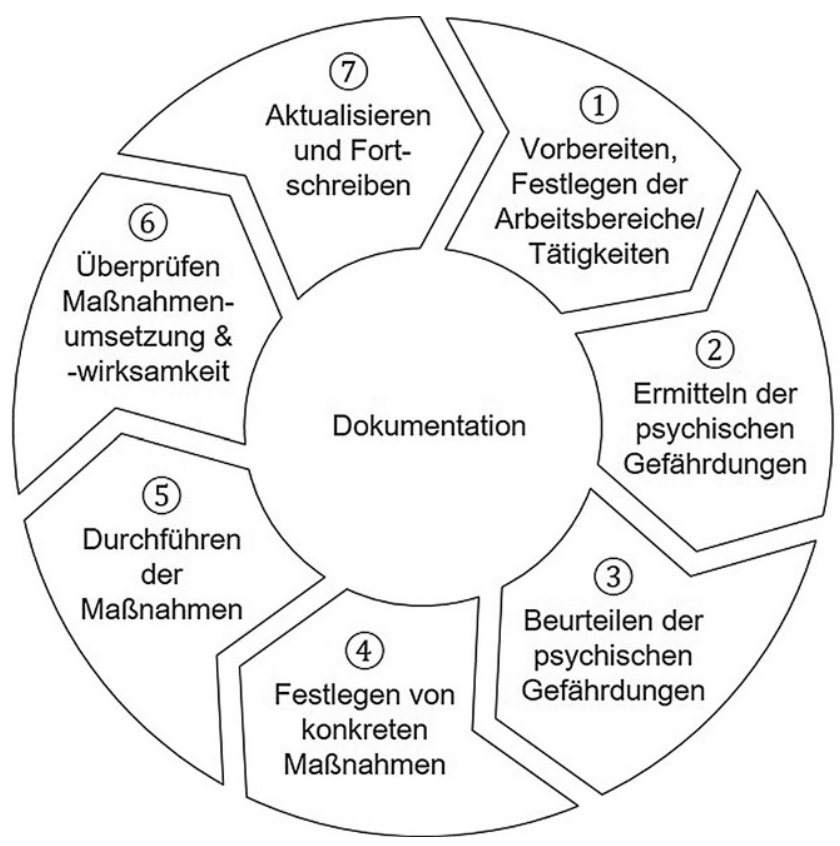

Abb. 2 Prozess der Gefährdungsbeurteilung psychischer Belastung. (In Anlehnung an BAuA 2020)

ratorinnen und Moderatoren besser in der Lage sind, mit Beschäftigten in Workshops realistische Lösungsvorschläge zu erarbeiten.

Wenn die Qualifizierung von Arbeitsschutz-Agierenden bzw. Moderatorinnen und Moderatoren im Betrieb nicht möglich ist, ist es anzuraten, externe Dienstleisterinnen oder Dienstleister einzuschalten, die die Gefährdungsbeurteilung mit entsprechender fachlicher Expertise planen und umsetzen.

\subsection{Nachhaltigkeit - Dranbleiben und Weitermachen}

In vielen Betrieben, in denen psychische Gefährdungen bereits ermittelt und beurteilt sowie erste Maßnahmen abgeleitet sind, wird die Gefährdungsbeurteilung als Chance für das Anstoßen betrieblicher Verbesserungsprozesse erkannt. Um Verbesserungen nachhaltig zu verankern, sind „Dranbleiben“ und „Weitermachen“" wesentliche Schlüsselfaktoren.

Dranbleiben Für eine erfolgreiche Gefährdungsbeurteilung ist es wichtig, dass alle Schritte mit dem erforderlichen Nachdruck und kontinuierlichem Engagement begleitet werden. Umstrukturierungen im Unternehmen, Personalwechsel oder das schlichte „Versinken im Tagesgeschäft" sollten nicht dazu führen, dass das Vorhaben der Gefährdungsbeurteilung psychischer Belastung stagniert oder gar völlig scheitert.
Der Gesetzgeber beschreibt in $\S 6$ Ziffer 1 ArbSchG, welche Inhalte zur Gefährdungsbeurteilung schriftlich dokumentiert werden müssen. Dazu zählen die Ergebnisse der Beurteilung der psychischen Gefährdungen, eine Aufstellung der festgelegten Maßnahmen sowie das Ergebnis der Kontrolle der Maßnahmenumsetzung. Es ist nicht ausreichend, nur die Gefährdungen zu ermitteln oder erste Maßnahmen festzulegen. Vielmehr ist die Gefährdungsbeurteilung psychischer Belastung ein Prozess, der von der Planung und Vorbereitung über die Analyse und Beurteilung der psychischen Gefährdung bis zur Festlegung, Durchführung und Überprüfung von Maßnahmen reicht. Daran schließt sich die Aktualisierung und Fortschreibung der Gefährdungsbeurteilung an (Abb. 2).

Für einen nachhaltigen Prozess der Gefährdungsbeurteilung ist es zunächst sinnvoll, dass ein schriftlicher Auftrag von der Geschäftsführung für die Gefährdungsbeurteilung vorliegt. Zudem muss gewährleistet sein, dass die erforderlichen Ressourcen sowohl für die Ermittlung der psychischen Belastung als auch für etwaige Maßnahmen zur Verbesserung der Arbeitsbedingungen bereitgestellt werden.

Der Prozess der Gefährdungsbeurteilung psychischer Belastung gerät in vielen Betrieben beim Prozessschritt der Maßnahmenumsetzung ins Stocken. Dies betrifft insbesondere größere, gegebenenfalls kostenintensivere Maßnahmen, die beispielsweise aufgrund von langen Kommunikations- und Entscheidungswegen sowie Ressourcenengpässen nicht umgesetzt werden. Das lange Aufschieben oder gar das Ausbleiben von entwickelten Maßnahmen kann zu Unmut und Frustration bei den Beschäftigten führen (z.B. „Mitarbeiterbefragung für die Schublade“, „,es passiert ja sowieso nichts"). Wenn aus den mithilfe der Beschäftigten gesammelten Informationen keine erkennbaren Konsequenzen folgen, nimmt die Bereitschaft der Beschäftigten $\mathrm{ab}$, sich an weiteren Befragungen oder Workshops zu beteiligen oder sich für Veränderungsprozesse im Betrieb $\mathrm{zu}$ engagieren. Generell gilt deshalb: Maßnahmen sollten zeitnah umgesetzt und kontrolliert sowie bezüglich ihrer Wirksamkeit überprüft werden.

Die Erfahrung zeigt, dass oftmals schon einfache Gestaltungsmaßnahmen bei den Arbeitsbedingungen zu einer für die Beschäftigten deutlich wahrnehmbaren Entlastung führen können (z.B. die Lautstärke von Werbemonitoren in Verkaufsräumen reduzieren, Informationsfluss verbessern durch Schichtübergabe-Bücher). Somit ist für die Beschäftigten erkennbar, dass die Ergebnisse der Gefährdungsbeurteilung nicht ,in der Schublade verschwinden“, sondern positive Veränderungen nach sich ziehen.

Wurden mehrere Problemfelder identifiziert, ist eine schrittweise Entwicklung und Umsetzung von Maßnahmen zu empfehlen. Schwerpunkte bzw. Prioritäten können beispielsweise nach Dringlichkeit oder Anzahl der betroffenen Beschäftigten gesetzt werden (GDA 2017b). Maßnahmen 
Tab. 1 Erfolgsbausteine für die Gefährdungsbeurteilung psychischer Belastung

\begin{tabular}{ll}
\hline Erfolgsbaustein & Kurzbeschreibung \\
\hline Organisation & $\begin{array}{l}\text { Die Gefährdungsbeurteilung ist fest in den Strukturen und Prozessen des betrieblichen Arbeits- und Gesundheitsschutzes ver- } \\
\text { ankert. Die Vorbereitung, Durchführung und Nachbereitung erfolgen systematisch und gut strukturiert. }\end{array}$ \\
& $\begin{array}{l}\text { Die erforderliche innerbetriebliche Akzeptanz wird durch eine breite und kontinuierliche Beteiligung von Beschäftigten, Füh- } \\
\text { rungskräften und verschiedenen Arbeitsschutz-Agierenden (z. B. Arbeitsmedizinerinnen und -medizinern, Fachkräften für }\end{array}$ \\
& $\begin{array}{l}\text { Arbeitssicherheit, Betriebsrat/Personalrat) an der Planung, Durchführung, Ableitung und Kontrolle von Verbesserungsmaßnah- } \\
\text { men gesichert. }\end{array}$ \\
Kommunikation & $\begin{array}{l}\text { Alle Beteiligten werden angemessen und frühzeitig über Anlass, Ziel, Verlauf und Ergebnisse der Gefährdungsbeurteilung } \\
\text { psychischer Belastung informiert. Es besteht des Weiteren die Möglichkeit für einen kontinuierlichen innerbetrieblichen Aus- } \\
\text { tausch. }\end{array}$ \\
& $\begin{array}{l}\text { Die verantwortlichen und beteiligten Personen besitzen die erforderlichen Kompetenzen oder werden dazu mittels Schulun- } \\
\text { gen befähigt, die psychische Belastung in der Gefährdungsbeurteilung zu berücksichtigen. Alle besitzen ein gemeinsames }\end{array}$ \\
& $\begin{array}{l}\text { Verständnis über Ziel und Vorgehen bei der Gefährdungsbeurteilung psychischer Belastung. } \\
\text { Nachhaltigkeit }\end{array}$ \\
& $\begin{array}{l}\text { Alle Schritte der Gefährdungsbeurteilung werden mit dem erforderlichen Nachdruck und kontinuierlichem Engagement durch- } \\
\text { geführt. Die Gefährdungsbeurteilung psychischer Belastung wird als ein fester Regelprozess im Betrieb verankert. }\end{array}$ \\
\hline
\end{tabular}

können anschließend nach kurz-, mittel- und langfristiger Umsetzung differenziert werden, wobei die Beschäftigten über die entsprechenden Umsetzungspläne auf dem Laufenden gehalten werden sollten.

Haben Unternehmen Verbesserungsmaßnahmen festgelegt, muss überprüft werden, ob sie auch wirklich umgesetzt werden und ob sich die Arbeitssituation nach der Umsetzung in gewünschter Weise verändert. Die Umsetzungs- und Wirksamkeitsprüfung wird von Arbeitsschutz-Agierenden in den Betrieben als essentiell benannt, um die Nachhaltigkeit der Gefährdungsbeurteilung psychischer Belastung abzusichern. Allerdings zeigen bisherige Erkenntnisse aus Praxisprojekten, dass die Maßnahmenkontrolle in nur wenigen Betrieben durchgeführt wird. Wichtig ist deshalb, dass die Verantwortung für diesen wichtigen Prozessschritt im Unternehmen von Beginn an klar geregelt ist (GDA 2017b).

In der betrieblichen Praxis hat sich gezeigt, dass eine Wirksamkeitskontrolle durch betriebsexterne Personen von Vorteil sein kann (aus der Unternehmenszentrale), weil dadurch ein „Zurückfallen in alte Muster" vermieden wird. Betriebliche Agierende berichten außerdem, dass vor allem Beschäftigte eine externe Kontrolle bevorzugen. Im Vergleich zu internen Kontrollierenden (z.B. Betriebsratsmitgliedern) werden externe Kontrollierende von Beschäftigten als stärker einflussnehmend wahrgenommen (,die Kontrolle hat ein anderes Gewicht“, Beschäftigte haben „,eher das Gefühl, dass sich etwas ändert"). Idealerweise erfolgt bei der Maßnahmenkontrolle und Wirksamkeitsprüfung eine Zusammenarbeit von internen und externen Kontrollierenden.

Zur Maßnahmenkontrolle und Wirksamkeitsprüfung können neben der Durchführung von Workshops auch Kurzbefragungen der Beschäftigten und Führungskräfte sowie Begehungen vorgenommen werden. Berücksichtigt werden sollte dabei, dass sich positive Auswirkungen nicht sofort, sondern auch erst mittel- oder langfristig zeigen können (GDA 2017b). Dies betrifft beispielsweise Verbes- serungen hinsichtlich der Mitarbeiter- und/oder Kundenzufriedenheit, die Senkung des Krankenstandes oder die Sensibilisierung der Führungskräfte (BAuA 2020).

Weitermachen Um die Gefährdungsbeurteilung in den betrieblichen Abläufen im Sinne eines Verbesserungsprozesses langfristig zu verankern, ist in den Betrieben eine Kultur der Prävention erforderlich, die Sicherheit und (psychische) Gesundheit bei der Arbeit in allen unternehmerischen Entscheidungen und Abläufen berücksichtigt (vgl. Kampagne „kommmitmensch“ der Berufsgenossenschaften, Unfallkassen und DGUV).

Sind psychische Gefährdungen fester Bestandteil der Präventionskultur eines Betriebs, erleichtert dies die Fortschreibung und Aktualisierung der Gefährdungsbeurteilung. Die Fortschreibung und Aktualisierung ist notwendig, wenn im Betrieb neue Gefährdungen auftreten, sich betriebliche Gegebenheiten hinsichtlich Sicherheit und Gesundheit verändern oder Gefährdungen neu erkannt wurden (BAuA 2020). Als konkrete Anlässe für eine erneute Ermittlung und Beurteilung von Tätigkeiten bzw. Arbeitsplätzen benennt die Bundesanstalt für Arbeitsschutz und Arbeitsmedizin (ebd.) unter anderem hohe Fehlzeiten aufgrund arbeitsbedingter Gesundheitsbeeinträchtigungen, Änderungen der Arbeitsorganisation und/oder Tätigkeitsabläufe, die Anschaffung neuer Arbeitsmittel, neue Informationen zum Arbeits- und Gesundheitsschutz oder Änderungen von Arbeitsschutzvorschriften. Auch das Auftreten eines Arbeitsoder Beinaheunfalls erfordert die Aktualisierung der Gefährdungsbeurteilung. Häufig werden bei (Beinahe-)Unfällen zunächst technische Ursachen untersucht oder ,menschliches Versagen" als Ursache angegeben. Jedoch ist eine Überprüfung der psychischen Arbeitsanforderungen in solchen Fällen dringend anzuraten. Beispielsweise kann ein ungünstig gestalteter Arbeitsablauf zu einem solch hohen Zeitdruck führen, dass Sicherheitsmaßnahmen ausgelassen 
werden müssen und die Wahrscheinlichkeit für Unfälle steigt.

Erfahrungen aus Praxisprojekten zeigen, dass die Fortschreibung der Gefährdungsbeurteilung psychischer Belastung - wie sie in den ,Sieben Schritten zur Gefährdungsbeurteilung" der BAuA (2020; Abb. 2) vorgesehen ist - in den Betrieben häufig noch aussteht. Betriebliche Agierende aus Unternehmen mit Filialstruktur sind zum Teil der Ansicht, dass Analyseergebnisse und Maßnahmen von einem Filialbetrieb, in dem die Gefährdungsbeurteilung bereits durchgeführt wurde, auf andere Standorte übertragen werden können. Eine solche Vorgehensweise ist jedoch nicht anzuraten. Neben etwaigen Ähnlichkeiten bestehen immer auch Unterschiede zwischen den Unternehmensbereichen (d.h. Standorten, Filialen, Geschäftsstellen o. Ä.) eines Gesamtunternehmens. Mögliche Unterschiede betreffen beispielsweise die sozialen Beziehungen (zwischen Kolleginnen und Kollegen und zu Führungskräften) und die Arbeitsumgebung (z. B. bauliche Gegebenheiten und Einrichtungen). Daher sollte jede Filiale mit den von den Beschäftigten dort ausgeübten Tätigkeiten im Rahmen der Gefährdungsbeurteilung psychischer Belastung gesondert betrachtet werden, um sinnvolle, passfähige Maßnahmen ableiten und umsetzen zu können.

Eine Gesamtbetriebsvereinbarung zur Erfassung der psychischen Belastung in der Gefährdungsbeurteilung kann der „Startschuss“ für die Fortschreibung der Gefährdungsbeurteilung in allen Bereichen des Unternehmens sein. Eine solche Betriebsvereinbarung schafft Verbindlichkeit zwischen der Arbeitgeber- und Arbeitnehmerseite und schreibt das weitere Vorgehen fest.

\section{Zusammenfassung und Fazit}

Die zunehmende Bedeutung der psychischen Anforderungen geht mit einem Handlungsbedarf zur gesundheitsgerechten Gestaltung von Arbeitsbedingungen mit Blick auf psychische Belastung einher. Um psychische Gefährdungen zu erkennen und entsprechende Maßnahmen abzuleiten, sind Arbeitgeberinnen und Arbeitgeber verpflichtet, eine Gefährdungsbeurteilung psychischer Belastung durchzuführen. Für die Umsetzung haben sich fünf Erfolgsbausteine in der Praxis bewährt (in Anlehnung an BGHW 2019; DIN EN ISO 9000 ff.; GDA 2018; Treier 2019; Tab. 1).

Die erfolgreiche Umsetzung der Gefährdungsbeurteilung psychischer Belastung bietet Betrieben die Chance, anderen Betrieben „eine Nasenlänge“ voraus zu sein und die Gesundheit sowie Leistungsfähigkeit der Beschäftigten auch in Zeiten des Wandels zu erhalten.

Funding Open Access funding provided by Projekt DEAL.
Open Access Dieser Artikel wird unter der Creative Commons Namensnennung 4.0 International Lizenz veröffentlicht, welche die Nutzung, Vervielfältigung, Bearbeitung, Verbreitung und Wiedergabe in jeglichem Medium und Format erlaubt, sofern Sie den/die ursprünglichen Autor(en) und die Quelle ordnungsgemäß nennen, einen Link zur Creative Commons Lizenz beifügen und angeben, ob Änderungen vorgenommen wurden.

Die in diesem Artikel enthaltenen Bilder und sonstiges Drittmaterial unterliegen ebenfalls der genannten Creative Commons Lizenz, sofern sich aus der Abbildungslegende nichts anderes ergibt. Sofern das betreffende Material nicht unter der genannten Creative Commons Lizenz steht und die betreffende Handlung nicht nach gesetzlichen Vorschriften erlaubt ist, ist für die oben aufgeführten Weiterverwendungen des Materials die Einwilligung des jeweiligen Rechteinhabers einzuholen.

Weitere Details zur Lizenz entnehmen Sie bitte der Lizenzinformation auf http://creativecommons.org/licenses/by/4.0/deed.de.

\section{Literatur}

BAuA (2019) Arbeitswelt im Wandel. Zahlen - Daten - Fakten. https://www.baua.de/DE/Angebote/Publikationen/Praxis/A100. pdf?_blob=publicationFile\&v=6. Zugegriffen: 6. Jan. 2020

BAuA (2020) Sieben Schritte zur Gefährdungsbeurteilung. https://www.baua.de/DE/Themen/Arbeitsgestaltung-imBetrieb/Gefaehrdungsbeurteilung/Grundlagenwissen/SiebenSchritte-zur-Gefaehrdungsbeurteilung/Sieben-Schritte-zurGefaehrdungsbeurteilung_node.html. Zugegriffen: 7. Jan. 2020

Beck D, Lenhardt U (2019) Consideration of psychosocial factors in workplace risk assessments: findings from a company survey in Germany. Int Arch Occup Environ Health 92(3):435-451

BGHW (2019) Das PegA-Programm - Psychische Belastung in der Gefährdungsbeurteilung. Berufsgenossenschaft Handel und Warenlogistik. BGHW, Mannheim

Böckelmann I, Seibt R (2011) Methoden zur Indikation vorwiegend psychischer Berufsbelastung und Beanspruchung - Möglichkeiten für die betriebliche Praxis. Z Arb Wiss 65:205-221

Bräunig D, Kohstall T (2013) DGUV Report 1/2013. Berechnung des internationalen „Return on Prevention“ für Unternehmen: Kosten und Nutzen von Investitionen in den betrieblichen Arbeits- und Gesundheitsschutz. Deutsche Gesetzliche Unfallversicherung, Berlin

Debitz U, Buruck G, Mühlpfordt S, Muzykorska E, Lübbert U, Schmidt H (2014) Der Leitfaden zum Screening Gesundes Arbeiten (SGA). Physische und psychische Gefährdungen erkennen gesünder arbeiten! Version 3.0. Bundesanstalt für Arbeitsschutz und Arbeitsmedizin, Dortmund

DGUV (2019) Arbeitsunfallgeschehen 2018. Deutsche Gesetzliche Unfallversicherung, Berlin

Eckert R (2017) Hyperwettbewerb und Digitalisierung. In: Eckert R (Hrsg) Business Innovation Management. Gabler, Wiesbaden, S 1-14

Eichhorn D, Schuller K (2017) Gefährdungsbeurteilung psychischer Belastung - Reine Pflichterfüllung oder Nutzen für die Betriebe? Sich Ist Sich 68(10):428-433

EO-Institut (2015) Gefährdungsbarometer ${ }^{\circledR}$-Studie 2016 - Stand der Gefährdungsbeurteilung psychischer Belastung in der Praxis. https://www.eo-institut.de/wp-content/uploads/2018/04/GB_ Studie_2016_EO-Institut.pdf. Zugegriffen: 10. Dez. 2019

European Agency for Safety and Health at Work (2016) Second European Survey of Enterprises on New and Emerging Risks (ESENER-2). Overview report: Managing safety and health at work. Publications Office of the European Union, Luxembourg

GDA (2016) Empfehlungen zur Qualifizierung betrieblicher Akteure für die Umsetzung der Gefährdungsbeurteilung psychischer Belastung. http://www.gda-psyche.de/SharedDocs/Publikationen/ 
DE/broschuere-empfehlung-gefaehrdungsbeurteilung.pdf? blob=publicationFile \&v=11. Zugegriffen: 17. Jan. 2020

GDA (2017a) Leitlinie Organisation des betrieblichen Arbeitsschutzes. https://www.gda-portal.de/DE/Downloads/pdf/LeitlinieArbeitsschutzorganisation.pdf?_blob=publicationFile \&v=2. Zugegriffen: 17. Jan. 2020

GDA (2017b) Empfehlungen zur Umsetzung der Gefährdungsbeurteilung psychischer Belastung. http://www.gda-psyche. de/SharedDocs/Publikationen/DE/broschuere-empfehlunggefaehrdungsbeurteilung.pdf?_blob=publicationFile \&v=11. Zugegriffen: 17. Jan. 2020

GDA (2018) Leitlinie Beratung und Überwachung bei psychischer Belastung am Arbeitsplatz. http://www.gda-portal.de/DE/Downloads/ pdf/Leitlinie-Psych-Belastung.pdf?_blob=publicationFile. Zugegriffen: 4. Dez. 2019

Geschäftsstelle der Nationalen Arbeitsschutzkonferenz (2016) GDABetriebs- und Beschäftigtenbefragung: Grundauswertung der Betriebsbefragung 2015 und 2011 - betriebsproportional gewichtet. Bundesanstalt für Arbeitsschutz und Arbeitsmedizin, Berlin

GfA (2016) Selbstverständnis der Gesellschaft für Arbeitswissenschaft e. V. (GfA). http://www.gesellschaft-fuer-arbeitswissenschaft.de/ inhalt/dokumente/gfa-selbstverstaendnis-2016.pdf. Zugegriffen: 16. Dez. 2019

Hacker W (2018a) Menschengerechtes Arbeiten in der digitalisierten Welt. Eine wissenschaftliche Handreichung. Schriftenreihe Mensch - Technik - Organisation, Bd. 49. vdf Hochschulverlag, Zürich

Hacker W (2018b) Kooperative Arbeitsgestaltung durch Arbeitsplatzinhaber/innen - ein Lernprozess. Arbeit 2(4):391-400

Hacker W, Sachse P (2014) Allgemeine Arbeitspsychologie - Psychische Regulation von Tätigkeiten. Hogrefe, Göttingen

Hofmann M (2014) Gefährdungsbeurteilung durch den Arbeitgeber bezüglich psychischer Belastungen am Arbeitsplatz - Ein innereuropäischer Vergleich. Deutsche Gesellschaft für Psychiatrie und Psychotherapie, Psychosomatik und Nervenheilkunde (DGPPN) - Presse- und Öffentlichkeitsarbeit, Berlin. https://www.dgppn.de/_Resources/Persistent/ e3b006f945c8194e35063227ad1e2280bcc0346d/2014-07-2014Studie_LANG_Gefährdungsbeurteilung.pdf. Zugegriffen: 9. Jan. 2020

Hubrich A (2011) Psychische Gesundheitsrisiken in der Arbeit mit Bürgerkontakt. DGUV Forum 9(11):18-19

iga - Initiative Gesundheit und Arbeit (2015) iga.Report 28 - Wirksamkeit und Nutzen betrieblicher Prävention. https://www.igainfo.de/fileadmin/redakteur/Veroeffentlichungen/iga_Reporte/ Dokumente/iga-Report_28_Wirksamkeit_Nutzen_betrieblicher_ Praevention.pdf. Zugegriffen: 18. Dez. 2019

Lenhardt U (2017) Psychische Belastung in der betrieblichen Praxis - Erfahrungen und Sichtweisen präventionsfachlicher Berater. $\mathrm{Z}$ Arb Wiss 71:6-13

Lohmann-Haislah A (2012) Stressreport Deutschland 2012 - Psychische Anforderungen, Ressourcen und Befinden. Bundesanstalt für Arbeitsschutz und Arbeitsmedizin, Dortmund. https://www. baua.de/DE/Angebote/Publikationen/Berichte/Gd68.pdf\%3F blob\%3DpublicationFile\%26v\%3D4. Zugegriffen: 17. Dez. 2019

Looks P, Gilbert K, Roitzsch K, Hacker W (2011) Gefährdungsbeurteilung bei Verkaufspersonal. Pilotvalidierung der Tätigkeitsanalyse distributiver Dienstleistungen (TA-DD). Praev Gesundheitsf 4(4):238-244

Mühlpfordt S, Richter P (2003) Evaluation eines orientierenden Verfahrens zur Erfassung psychischer Belastungen am Arbeitsplatz. Schriftenreihe der Bundesanstalt für Arbeitsschutz und Arbeitsmedizin. Wissenschaftsverlag NW, Bremerhaven

Neuner R (2019) Die Gefährdungsbeurteilung psychischer Belastung bei der Arbeit. In: Neuner R (Hrsg) Psychische Gesundheit bei der Arbeit: Gefährdungsbeurteilung und Betriebliches Gesundheitsmanagement, S 43-101

Niedhammer I, Chastang J-F, Sultan-Taïeb H, Vermeylen G, ParentThirion A (2013) Psychosocial work factors and sickness absence in 31 countries in Europe. Eur J Public Health 23:622-629

Paridon H (2016) Psychische Belastung in der Arbeitswelt - Eine Literaturanalyse zu Zusammenhängen mit Gesundheit und Leistung Initiative Gesundheit \& Arbeit - Report 32. Initiative Gesundheit und Arbeit, Berlin

Rau R, Buyken D (2015) Der aktuelle Kenntnisstand über Erkrankungsrisiken durch psychische Arbeitsbelastungen: Ein systematisches Review über Metaanalysen und Reviews. Z Arb Organ 59(3):113-129

Rau R, Henkel D (2013) Zusammenhang von Arbeitsbelastungen und psychischen Erkrankungen - Review der Datenlage. Nervenarzt 84:791-798

Rothe I, Adolph L, Beermann B, Schütte M, Windel A, Grewer A, Lenhardt U, Michel J, Thomson B, Formazin M (2017) Psychische Gesundheit in der Arbeitswelt - Wissenschaftliche Standortbestimmung. Bundesanstalt für Arbeitsschutz und Arbeitsmedizin, Dortmund, Berlin, Dresden

Schmidt C, Pietrzyk U, Burisch M, Mußlick S, Hoffmann S (2012) Psychische Belastung in der Dienstleistungsbranche. Bundesanstalt für Arbeitsschutz und Arbeitsmedizin, Dortmund

Schütte M, Rothe I (2018) Prävention und Arbeitsgestaltung - Psychische Gesundheit in der Arbeitswelt. Arbeitsmed Sozialmed Umweltmed 53(Sonderheft):6-8

Stab N, Hacker W (2016) Stationsorganisation analysieren, bewerten und gestalten. Interaktive Vorlagen zur Bearbeitung des Verfahrens „Gute Stationsorganisation“. Bundesanstalt für Arbeitsschutz und Arbeitsmedizin, Dortmund

Treier M (2019) Gefährdungsbeurteilung psychischer Belastungen Begründung, Instrumente, Umsetzung. Springer Gabler, Wiesbaden

Zink KJ, Bosse CK (2019) Megatrends im Kontext von Arbeit und Organisation im 21. Jahrhundert. In: Zink KJ (Hrsg) Arbeit und Organisation im digitalen Wandel. Nomos, Baden-Baden, S 35-52 